\title{
PARAMETRIC PHONON-HELICON INTERACTION IN CENTROSYMMETRIC CRYSTALS WITH STRAIN-DEPENDENT DIELECTRIC CONSTANT
}

\author{
O.L. ARtemenko \\ Institute of Applied Pliysics, Academy of Sciences of Belarus \\ F. Skariny Str. 16, Minsk, 220072, Belarus \\ AND B.B. SevruK \\ Institute of Physics, Academy of Sciences of Belarus \\ F. Skariny Str. 70, Minsk, 220072, Belarus
}

(Received May 15, 1995; revised version February 27, 1996)

\begin{abstract}
The process of interaction between circularly polarized coherent transverse phonons and slow electrokinetic waves in the centrosymmetric crystal placed in a longitudinal magnetic field and possessing strong strain dependence of the dielectric constant caused by the electrostriction is considered. The cut-off system of equations for the process of nonlinear four-wave interaction in crystal with a quadratic electrostriction corresponding to direct energy conversion from helicon to acoustic wave is derived. The solution is sought for numerically in the vicinity of a synchronism point determined by the intersection of helicon and plionon dispersion curves at different values of semiconductor plasma parameters. The results show the possibility of effective phonon parametric amplification in semiconductor crystal with the electroinduced elastic anisotropy.
\end{abstract}

PACS numbers: $63.20 . \mathrm{Kr}$

\section{Introduction}

The processes of interaction of electromagnetic radiation with solid state plasma provide extremely important information on the fundamental properties of solids. The investigations on this subject cover a great number of problems, among which studying the transverse phonon-helicon interaction in piezoelectric semiconductors has been one of the most active frontiers in acoustoelectronics due to considerable potentialities in semiconductor plasma diagnostics [1-3]. Various aspects of the process of propagation and amplification of acoustohelicon waves in 
piezoelectrics (like $\mathrm{InSb}$ ) in low electric fields without account for collisional effects in plasma have been studied in different approximations in [4-7]. Recently several authors have taken into consideration hot carrier effects, temperature dependence of the collision frequency and nonlinearities in electron effective mass and collision frequency within the models with carriers drift [8-10]. One of the latest reports on phonon-helicon interaction in piezoelectric semiconductors [11] deals with the investigation of second-order nonlinear susceptibility arising due to parametric phonon-helicon interaction in a longitudinally magnetized noncentrosymmetric semiconductor. The resulted parametric amplification of the acoustohelicon modes was reported. The nonlinearities taken into account were electronic nonlinearity due to nonlinear current density and nonlinear polarization resulting from the effective nonlinear susceptibility of the medium.

The present paper deals with the investigation of phonon-helicon interaction in centrosymmetric crystal with a quadratic electrostriction nonlinearity. Centrosymmetric crystals with a high value of dielectric constant $\left(\varepsilon \approx 10^{3}\right)$ are known to exhibit reducing of their symmetry centre in an external electric field. This process is accompanied by appearance of a strong electromechanical coupling proportional to the square of field strength. In this case, elastic anisotropy and piezoelectric effect induced by the electric field and the electron-phonon interaction are caused by strain dependence of dielectric constant (SDDC) due to electrostriction [12]. The results obtained previously show the possibility of application of crystals with SDDC both for development of structures with an artificial helical elastic anisotropy and for effective amplification and generation of acoustic waves (AWs) in the process of interaction either with the electric field, which induces rotating acoustic anisotropy [12], or with the longitudinal electrokinetic waves (EKWs) [13]. However, it appears from the available literature that no attempt has been made to study the process of transverse phonon-helicon interaction in a longitudinally magnetized semiconductor crystals with SDDC.

In the present study we consider the nonlinear interaction between coherent transverse phonons and slow EKW (helicon) in a centrosymmetric semiconductor with SDDC placed in a longitudinal magnetic field. It is shown that a particular kind of rotating helical structure of elastic and dielectric properties is formed in the initially isotropic medium in the process of interaction between circularly polarised (CP) modes. We derived the cut-off system of equations for the process of four-wave interaction, the solution of which is sought for numerically in the vicinity of a synchronism point determined by the intersection of helicon and phonon dispersion curves, and studied the peculiarities of direct energy conversion from the EKWs to $\mathrm{AW}$ for $\mathrm{BaTiO}_{3}$ crystal at $300 \mathrm{~K}$.

\section{Acousto- and electroinduced anisotropy at the phonon-helicon interaction}

The propagation of the EKWs and AW in semiconductor crystal with SDDC in a magnetic field $B_{0}$ is described by the following equations of electrodynamics, theory of elasticity and charge transfer [2]:

$$
\nabla \times \nabla \times E+\frac{1}{c^{2}} \frac{\partial^{2} D}{\partial t^{2}}+\mu_{0} e N_{0} \frac{\partial^{2} V}{\partial t}=0
$$




$$
\begin{aligned}
& \frac{\partial \sigma_{i k}}{\partial x_{k}}=\rho \frac{\partial^{2} U_{i}}{\partial t^{2}}, \\
& \frac{\partial V}{\partial t}=-\frac{e}{m^{*}}\left[E+\left(V \times B_{0}\right)\right]-\nu V-\frac{\kappa_{\mathrm{B}} T}{m^{*} N_{0}} \nabla n, \\
& \frac{\partial n}{\partial t}=N_{0}(\nabla V), \\
& (\nabla D)=-e n,
\end{aligned}
$$

where $\rho$ is the crystal density, $\nu$ - collision frequency, $V$ - electron drift velocity in the field $E, m^{*}-$ electron effective mass. The relationships between the components of stress tensor $\sigma_{i k}$, induction vector $D_{i}$ and components of the tensor of elastic deformations $U_{i k}$ and electric field $E_{i}$ have the following form [14]:

$$
\begin{aligned}
\sigma_{i k} & =C_{i k l m}^{\mathrm{s}} U_{l m}+\frac{1}{2} \varepsilon_{0} g_{i k l m} E_{l} E_{m}-\varepsilon_{0} d_{l m i k r s} E_{l} E_{m} U_{r s}, \\
D_{i} & =\varepsilon_{0}\left(\varepsilon_{i k} E_{k}-g_{i k l m} E_{k} U_{l m}+d_{i k l m r s} E_{k} U_{r s} U_{l m}\right),
\end{aligned}
$$

where $g$ and $d$ are linear and quadratic electrostriction tensors; $\varepsilon_{0}, \varepsilon$-vacuum permittivity and dielectric constant of the lattice, respectively; $C^{\text {s }}$ - elastic moduli.

Let a shear AW $U=\left(U_{2}, U_{3}\right) \sim \exp [\mathrm{i}(k x-\omega t)]$ propagate along the $x$-axis of the crystal with SDDC. The magnetic field $B_{0}$ is applied in the same direction and the helicon mode $E=\left(E_{2}, E_{3}\right) \sim \exp [(k x-\Omega t)]$ is excited. Then Eq. (1) with regard to (3) and (7), and Eq. (2) with regard to (4)-(6) could be reduced to a system of two nonlinear equations

$$
\begin{aligned}
& \frac{\partial^{2} E}{\partial x^{2}}-\frac{1}{c^{2}} \frac{\partial^{2}}{\partial \iota^{2}}\left\{\left[\widehat{\varepsilon}_{\mathrm{p}}+\Delta \widehat{\varepsilon}\left(U^{2}\right)\right] E\right\}=0, \\
& \rho \frac{\partial^{2} U}{\partial \iota^{2}}-\frac{\partial}{\partial x}\left\{\left[C_{\mathrm{s}}+\Delta \widehat{C}_{\mathrm{s}}\left(E^{2}\right)\right] \frac{\partial U}{\partial x}\right\}+\operatorname{egn} E=0,
\end{aligned}
$$

where $\widehat{\varepsilon}_{\mathrm{p}}(\Omega)$ - tensor of the lattice dielectric constant of semiconductor in the magnetic field [2], $\Delta \widehat{\varepsilon}\left(U^{2}\right)$ and $\Delta \widehat{C}_{\mathrm{s}}\left(E^{2}\right)$ are acoustic field induced additional terms determined by the quadratic electrostriction, which components for the cubic crystal are equal to

$$
\begin{aligned}
& \varepsilon_{22}^{\mathrm{p}}=\varepsilon_{33}^{\mathrm{p}}=\frac{\varepsilon \omega_{\mathrm{p}}^{2}(\Omega-\mathrm{i} \nu)}{\Omega\left[(\Omega-\mathrm{i} \nu)^{2}-\omega_{\mathrm{c}}^{2}\right]}, \\
& -\varepsilon_{23}^{\mathrm{p}}=\varepsilon_{32}^{\mathrm{p}}=\mathrm{i} \frac{\varepsilon \omega_{\mathrm{p}}^{2} \omega_{\mathrm{c}}}{\Omega\left[(\Omega-\mathrm{i} \nu)^{2}-\omega_{\mathrm{c}}^{2}\right]}, \\
& \Delta \varepsilon_{22}=\varepsilon\left(d_{1} U_{21}^{2}+d_{2} U_{31}^{2}\right), \quad \Delta \varepsilon_{33}=d_{2} U_{21}^{2}+d_{1} U_{31}^{2}, \\
& \Delta \varepsilon_{23}=\Delta \varepsilon_{32}=2 d_{3} U_{21} U_{31},
\end{aligned}
$$

$$
\Delta C_{22}=-\varepsilon \varepsilon_{0}\left[\left(g^{2}-d_{1}\right) E_{2}^{2}+d_{2} E_{3}^{2}\right], \quad \Delta C_{33}=-\varepsilon \varepsilon_{0}\left[\left(d_{2} E_{2}^{2}+\left(g^{2}-d_{1}\right) E_{3}^{2}\right],\right.
$$




$$
\Delta C_{32}=\Delta C_{23}=-\varepsilon \varepsilon_{0} E_{2} E_{3}\left(g^{2}-2 d_{3}\right),
$$

where $\omega_{\mathrm{c}}=e B_{0} / m^{*}, \omega_{\mathrm{p}}^{2}=N_{0} e^{2} / m^{*} \varepsilon \varepsilon_{0}-$ cyclotron and electron-plasma frequencies, respectively; $g=g_{66} \sim \varepsilon / 3, d_{1}=d_{155}, d_{2}=d_{144}, d_{3}=d_{456} \sim \varepsilon^{2} / 9$ [14].

The propagation of the AW in a crystal with SDDC is accompanied by longitudinal EKWs (space clarge waves), due to the piezoelectric effect, which is induced by the transverse electric field of the helicon. The Poisson equation (5) with regard to (7) yields the following expression for the piezoelectric field strength:

$$
\frac{\partial E_{1}}{\partial x}=-\frac{e n}{\varepsilon \varepsilon_{0}}+g \frac{\partial}{\partial x}\left(\frac{\partial U}{\partial x} E\right) \text {. }
$$

Starting from the equations of impulse conservation (3) and continuity (4) and taking into account Eq. (12) one can obtain the following formula for the piezoelectrically excited longitudinal plasma oscillations:

$$
\frac{\partial^{2} n}{\partial t^{2}}+\nu \frac{\partial n}{\partial t}-D_{1} \frac{\partial^{2} n}{\partial x^{2}}-g N_{0} \frac{e}{m} \frac{\partial}{\partial x}\left(\frac{\partial U}{\partial x} E\right)+\omega_{\mathrm{p}}^{2} n=0,
$$

where $D_{1}=k_{\mathrm{B}} T / \mathrm{m}^{*}, N_{0}$ being the equilibrium electron concentration and $n-$ the electron concentration perturbation. Assuming that $n \sim \exp [\mathrm{i}(k x-\omega t)]$, one can get from (13):

$$
n=\frac{e g N_{0}}{m Q(\omega, k)} \frac{\partial}{\partial x}\left(\frac{\partial U}{\partial x} E\right),
$$

where $Q(\omega, k)=-\omega^{2}-\mathrm{i} \omega \nu+\omega_{\mathrm{p}}^{2}\left(1+\lambda_{\mathrm{D}}^{2} k^{2}\right), \lambda_{\mathrm{D}}^{2}$ is the Debye screening length. Then the AW propagation equation (9) with regard to (14) may be written as

$$
\rho \frac{\partial^{2} U}{\partial t^{2}}=\frac{\partial}{\partial x}\left\{\left[C_{\mathrm{s}}+\Delta \widehat{C}_{\mathrm{s}}\left(E^{2}\right)-\frac{g^{2} \omega_{\mathrm{p}}^{2} \varepsilon \varepsilon_{0}}{Q(\omega, k)}(E \cdot E)\right] \frac{\partial U}{\partial x}\right\},
$$

where $(E \cdot E)$ is a dyad.

If the AW frequency $\omega \ll \omega_{\mathrm{p}}^{2}$ and $\lambda_{\mathrm{D}}^{2} k^{2} \ll 1$, then $\omega_{\mathrm{p}}^{2} / Q \approx 1+\mathrm{i} \omega / \omega_{\mathrm{M}}$, where $\omega_{\mathrm{M}}=\omega_{\mathrm{p}}^{2} / \nu-$ the Maxwell relaxation frequency. The influence of induced piezoelectric effect on the elastic moduli of the crystal in this case is screened by charge carriers and is reduced to the acoustoelectronic attenuation, which can be described by introducing the complex elastic moduli $C_{\mathrm{s}}^{\prime}+\mathrm{i} C_{\mathrm{s}}^{\prime \prime}$, where $C_{\mathrm{s}}^{\prime}=\rho V_{t}^{2}$, $V_{t}^{2}$ being the velocity of the transverse AW, $C_{\mathrm{s}}^{\prime \prime}=\alpha(E \cdot E), \alpha=\varepsilon \varepsilon_{0} g \omega / \omega_{\mathrm{M}}$ is the coefficient of acoustoelectronic damping of the $\mathrm{AW}$ in the process of interaction with longitudinal plasma oscillations.

Assuming that $\omega_{c} \gg \omega$, let us consider an interaction of a clockwise polarized helicon $E=E(x, t)(1,-\mathrm{i})+$ c.c. with the AW (counter-clockwise polarized mode is greatly damped in this case). If the AW is also clockwise polarized $U=U(x, t)(1,-\mathrm{i})+$ c.c., then neglecting the transformation of wave polarization in the process of nonlinear interaction, we obtain

$$
\begin{aligned}
& \Delta \widehat{C}_{\mathrm{s}}=-\frac{\varepsilon \varepsilon_{0}}{4}\left[2|E|^{2} \Delta_{1}+E^{2} \Delta_{2}\left(\begin{array}{rr}
1 & -\mathrm{i} A \\
-\mathrm{i} A & -1
\end{array}\right)+\text { c.c. }\right], \\
& \Delta \widehat{\varepsilon}=-\frac{\varepsilon}{4}\left[2\left|\frac{\partial U}{\partial x}\right|^{2} \Delta_{1}+\left(\frac{\partial U}{\partial x}\right)^{2} \Delta_{2}\left(\begin{array}{rr}
1 & -\mathrm{i} A \\
-\mathrm{i} A & -1
\end{array}\right)+\text { c.c. }\right],
\end{aligned}
$$


where $\Delta_{1}=d_{1}+d_{2}, \Delta_{2}=d_{1}-d_{2}$, and $A=2 d_{3} / \Delta_{2}-$ coefficient of induced anisotropy of the crystal. Thus, a helical rotating anisotropy of elastic and dielectric properties is induced in the initial medium at the process of wave interaction.

\section{Interaction of the CP AW with the helicon. Cut-off equations}

Let us consider a process of energy conversion from the helicon to the AW in agreement with the synchronism of a degenerated four-wave interaction $\omega+\omega=$ $\Omega+\Omega$ and $k+k=K+K+\Delta$, where $\Delta$ is a phase mismatch. Let $A \approx 1$, i.e. the initial medium is approximately transversely isotropic. Substituting $(10),(11)$ and (16) in Eqs. (8),(15) and taking into account the nonlinear terms $\sim|E|^{2} U, E^{2} U^{*}$, $|\partial U / \partial x|^{2} E,(\partial U / \partial x)^{2} E^{*}$, which contributc to direct energy conversion, we obtain a system of equations for the amplitudes of $\mathrm{CP}$ waves

$$
\begin{aligned}
& \rho \frac{\partial^{2} U}{\partial \iota^{2}}-\frac{\partial}{\partial x}\left[\left(C_{\mathrm{s}}-\Delta_{1} \frac{\varepsilon \varepsilon_{0}}{2}|E|^{2}\right) \frac{\partial U}{\partial x}+\Delta_{2} \frac{\varepsilon \varepsilon_{0}}{4} E^{2} \frac{\partial U^{*}}{\partial x}\right]+\text { c.c. }=0 \\
& \frac{\partial^{2} E}{\partial x^{2}}-\frac{1}{c^{2}} \frac{\partial^{2}}{\partial t^{2}}\left\{\left[\left(\varepsilon_{22}^{\mathrm{p}}-\varepsilon_{32}^{\mathrm{p}}\right)+\Delta_{1} \frac{\varepsilon}{2}\left|\frac{\partial U}{\partial x}\right|^{2}\right] E+\Delta p_{2} \frac{\varepsilon}{4}\left(\frac{\partial U}{\partial x}\right)^{2} E^{*}\right\} \\
& \quad+\text { c.c. }=0 .
\end{aligned}
$$

In the approximation of slowly varying amplitudes $U=\bar{U}(x) \exp [\mathrm{i}(k x-\omega t)]$, $E=\bar{E}(x) \exp [\mathrm{i}(k x-\Omega t)]$ the system $(17),(18)$ takes the form

$$
\begin{aligned}
& -2 \mathrm{i} C_{\mathrm{s}} k \frac{\partial \bar{U}}{\partial x}+\Delta_{2} \frac{\varepsilon \varepsilon_{0}}{4} \bar{E}^{2} \bar{U}^{*} \exp (\mathrm{i} \Delta x)+\bar{U}\left[-\rho \omega^{2}+k^{2}\left(C_{\mathrm{s}}-\Delta_{1} \frac{\varepsilon \varepsilon_{0}}{2}|\bar{E}|^{2}\right)\right] \\
& \quad+\text { c.c. }=0, \\
& -2 \mathrm{i} K \frac{\partial \bar{E}}{\partial x}-k^{2} \Delta_{2} \frac{\varepsilon \Omega^{2}}{4 c^{2}} \bar{E}^{*} \bar{U}^{2} \exp (-\mathrm{i} \Delta x)+\bar{E}\left[\varepsilon_{\mathrm{p}} \frac{\Omega^{2}}{c^{2}}-K^{2}\left(1-\Delta_{1} \frac{\varepsilon \Omega^{2}}{2 c^{2}}|\bar{U}|^{2}\right)\right] \\
& \quad+\text { c.c. }=0,
\end{aligned}
$$

where $\varepsilon_{\mathrm{p}}=\varepsilon_{22}^{\mathrm{p}}-\varepsilon_{32}^{\mathrm{p}}=\varepsilon_{\mathrm{p}}^{\prime}+\mathrm{i} \varepsilon_{\mathrm{p}}^{\prime \prime}, C_{\mathrm{s}}=C_{\mathrm{s}}^{\prime}+\mathrm{i} C_{\mathrm{s}}^{\prime \prime}$.

Assuming that for noncoupled waves with initial amplitudes $\bar{U}_{0}, \bar{E}_{0}$ the following clispersion relations are' valid:

$$
\begin{aligned}
& \rho \omega^{2}+k^{2}\left(C_{\mathrm{s}}^{\prime}-\Delta_{1} \frac{\varepsilon \varepsilon_{0}}{2} \bar{E}_{0}^{2}\right)=0, \\
& \varepsilon_{\mathrm{p}}^{\prime}(\Omega) \frac{\dot{\Omega}^{2}}{c^{2}}-K^{2}\left(1-\Delta_{1} \frac{\varepsilon \Omega^{2}}{2 c^{2}} \bar{U}_{0}^{2}\right)=0,
\end{aligned}
$$

one can find resonance values $\omega \approx \Omega=\omega_{\text {res }}, k \approx K=k_{\text {res }}$ corresponding to the intersection point of dispersion curves. Here the resonance frequency $\omega_{\text {res }}$ is determined by the following expression: $\omega_{\text {res }} \approx \omega_{\mathrm{p}}^{2} \omega_{\mathrm{c}} V_{t}^{2} \varepsilon /\left(\omega_{\mathrm{c}}^{2}+\nu^{2}\right) c^{2}$, at $\omega \ll \omega_{\mathrm{c}}$. Making a transition to real amplitudes and phases in (19) $\bar{E}(x)=A_{1}(x) \exp \left[i \psi_{1}(x)\right], \bar{U}(x)=A_{2}(x) \exp \left[\mathrm{i} \psi_{2}(x)\right]$, we obtain the final system of 
equations (we neglect the influence of $\bar{U}_{0}$ and $\bar{E}_{0}$ amplitude changes on variation of wave phase velocities):

$$
\begin{aligned}
& \frac{\partial A_{1}}{\partial x}+\alpha_{1} A_{1}+\beta_{1} \Delta_{2} A_{2}^{2} A_{1} \sin \Psi=0, \\
& \frac{\partial A_{2}}{\partial x}+\alpha_{2} A_{2}-\beta_{2} \Delta_{2} A_{1}^{2} A_{2} \sin \Psi=0, \\
& \frac{\partial \Psi}{\partial x}-\Delta+\frac{\Delta_{2}}{4}\left(\beta_{1} A_{2}^{2}-\beta_{2} A_{1}^{2}\right) \cos \Psi=0,
\end{aligned}
$$

where $\Psi=2\left(\psi_{1}-\psi_{2}\right)+\Delta x-$ generalized phase, $\alpha_{1}=-\varepsilon_{\mathrm{p}}^{\prime \prime} \Omega^{2} / 2 c^{2} K^{\prime \prime}$, and $\alpha_{2}=-C_{\mathrm{s}}^{\prime \prime} k / 2 C_{\mathrm{s}}^{\prime}$ - attenuation coefficients, $\beta_{1}=\varepsilon \Omega^{2} k / c^{2}, \beta_{2}=\varepsilon \varepsilon_{0} k / C_{\mathrm{s}}^{\prime}$.

\section{Numerical results and discussion}

The system of Eqs. (21) was solved numerically at different values of semiconductor plasma parameters $\omega_{\mathrm{p}}, \omega_{\mathrm{c}}$ and $\nu$, initial amplitudes $\bar{U}_{0}, \bar{E}_{0}$ and optimal values of phase mismatch $\Delta=0$ and generalized phase $\Psi=\pi / 2$. As a typical case, the calculations were performed for the $\mathrm{BaTiO}_{3}$ cubic crystal at $300 \mathrm{~K}$ with the following physical constants: $N_{0}=10^{23} \mathrm{~m}^{-3}, m=0.0145 m_{0}, \varepsilon=2 \times 10^{3}$, $\rho=4 \times 10^{3} \mathrm{~kg} \mathrm{~m}^{-3}, V_{t}=2.5 \times 10^{3} \mathrm{~s}^{-1}, m_{0}$ being the free electron mass. The initial values of the AW power $P_{0}$ and electromagnetic field amplitude $E_{0}$ are assumed to be equal to $0.01 \mathrm{~W} \mathrm{~cm}^{-2}$ and $25 \mathrm{kV} \mathrm{cm}^{-1}$, accordingly. Calculated dependences of the normalized AW amplitude $A_{2}$ from interaction length $z$ without regard for helicon attenuation are shown in Fig. 1. A full mutual energy conversion from the EKW to $\mathrm{AW}$ modes takes place. Ilowever, the process of wave interaction greatly depends on the collisional attenuation of the helicon. Such a situation is

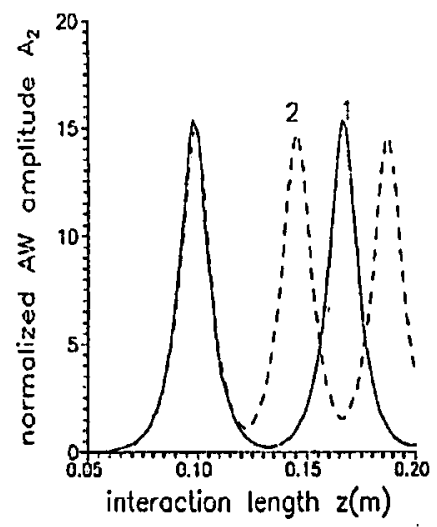

Fig. 1. Variation of the normalized $A W$ amplitude with interaction length in the absence of helicon attenuation at $\omega_{\mathrm{p}}=3.3 \times 10^{12} \mathrm{~s}^{-1}, \omega_{\mathrm{c}}=1 \times 10^{12} \mathrm{~s}^{-1}$ and $\nu\left(\mathrm{s}^{-1}\right)$ : $1-0,2-1 \times 10^{8}$. 


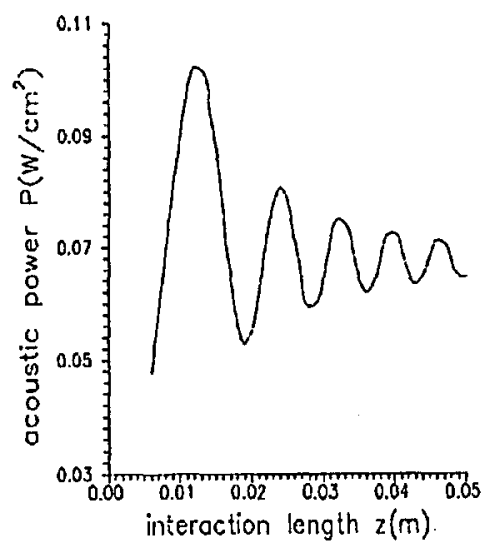

Fig. 2. Variation of the $A W$ power with interaction length at $\omega_{\mathrm{p}}=3.3 \times 10^{12} \mathrm{~s}^{-1}$, $\omega_{c}=1 \times 10^{12} \mathrm{~s}^{-1}, \omega_{\text {res }}=1.5 \times 10^{6} \mathrm{~s}^{-1}, \nu=5 \times 10^{10} \mathrm{~s}^{-1}$.

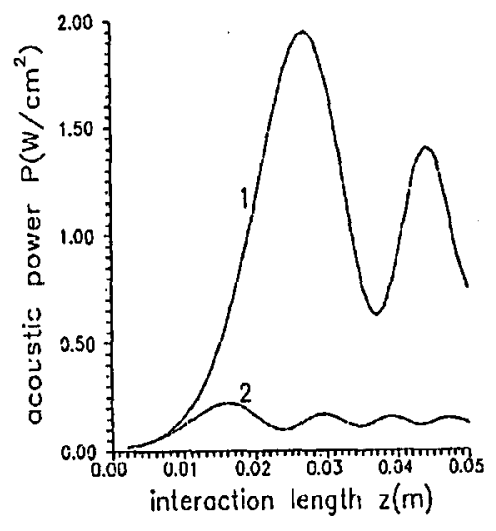

Fig. 3. Variation of the AW power with interaction length for different collision frequency $\nu\left(\mathrm{s}^{-1}\right): 1-1 \times 10^{10} \mathrm{~s}^{-1}, 2-3 \times 10^{10} \mathrm{~s}^{-1}$ and $\omega_{\mathrm{p}}=3.3 \times 10^{12} \mathrm{~s}^{-1}$, $\omega_{\mathrm{c}}=1 \times 10^{12} \mathrm{~s}^{-1}, \omega_{\text {res }}=1.5 \times 10^{6} \mathrm{~s}^{-1}$.

shown in Fig. 2, where the variation of the AW power $P$ with interaction length $z$ is presented for the collision frequency $\nu=5 \times 10^{10} \mathrm{~s}^{-1}\left(\omega_{\mathrm{p}}=3.3 \times 10^{12} \mathrm{~s}^{-1}\right.$, $\left.\omega_{\mathrm{c}}=1 \times 10^{12} \mathrm{~s}^{-1}\right)$. The curve has an oscillating character with a pronounced damping of the mutual energy conversion between different modes resulting from energy exhaustion of the electromagnetic pump wave. However, the attained AW power level is maintained. The generation of the AW takes place at resonance frequency $\omega_{\text {res }} \approx 1.5 \times 10^{6} \mathrm{~s}^{-1}$ determined from Eq. (19). As can be seen from Fig. 3, where variations of the AW power with interaction length are plotted for two different values of the collision frequency $\nu=1 \times 10^{10}$ and $3 \times 10^{10} \mathrm{~s}^{-1}$, the acoustic power of the generated AW decreases with increase in $\nu$.

The process of amplification of the AW has also been numerically analyzed at different values of the cyclotron frequency $\omega_{c}$ (Fig. 4). It could be seen that 


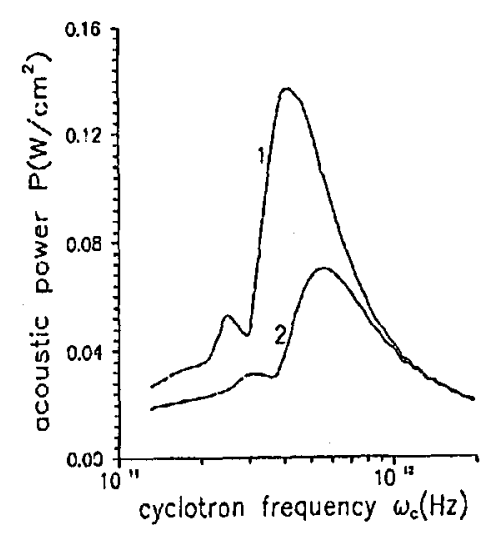

Fig. 4. Variation of the AW power witl cyclotron frequency at $\omega_{\mathrm{p}}=3.3 \times 10^{12} \mathrm{~s}^{-1}$, $\omega_{\text {res }}=1.5 \times 10^{6} \mathrm{~s}^{-1}$, and $\nu\left(\mathrm{s}^{-1}\right): 1-1 \times 10^{10} \mathrm{~s}^{-1}, 2-3 \times 10^{10} \mathrm{~s}^{-1}$.

the AW power $P$ increases initially with $\omega_{\mathrm{c}}$ due to decrement in helicon attenuation [2]. Then the AW power attains its peak value and undergoes a progressive decrement. This fact could be attributed to a reduction in the magnitude of nonlinear coefficients $\beta_{1}, \beta_{2}$ from Eq. (21) due to lowering of $\omega_{\text {res }}$ value with increase in the cyclotron frequency $\omega_{c}$. The calculations also reveal that for the realization of the reverse process of helicon amplification by the $A W$, one should have materials with a relatively high dielectric constant $\varepsilon \approx 10^{4}$ or should decrease the collisional attenuation of the helicon.

\section{Conclusions}

The effect of resonance amplification of the AW takes place at frequencies corresponding to the ultrasound. Ilowever, by additional doping of the semiconductor one can substantially increase the plasma frequency and the resonance frequency of the $A W$ as well. It should be also mentioned that eflicient $A W$ generation could be reached at relatively low fields $\left(\approx 25 \mathrm{kV} \mathrm{cm}^{-1}\right)$ of the electromagnetic wave. The above discussion reveals that the amplification of the CP AW by electrokinetic modes in a longitudinally magnetized semiconductor plasma can be easily achieved in crystals with SDDC coupling with the reasonable power level of the AW (up to several $W \mathrm{~cm}^{-2}$ ). The effect could be observed in cubic ferroelectric semiconductors like $\mathrm{BaTiO}_{3}$ in paraphase or ferroelectric-based ceramics.

The helicon waves are known to be used for measuring the magnitude of magnetic fields [3], as their attenuation strongly depends on the cyclotron frequency value. The resonance nature of the phonon-helicon interaction considered in this paper offers some possibilities for measuring the magnetic fields by making use of the AW as a physical quantity, which is very sensitive to the magnitude of the cyclotron frequency. Unfortunately, it is extremely difficult to compare our results because of the nonavailability of relevant experimental data on the active phonon-helicon interaction in crystals with SDDC. However, it is reasonably safe to suggest that the effect of resonance interaction between acoustic and electromagnetic waves described above could be used both for ultrasonic generation 
and diagnostics of the electron plasma in semiconductors with a high dielectric constant.

\section{References}

[1] L. Solymer, C.N. Lashmore-Davies, Int. J. Electron. 22, 549 (1967).

[2] M.C. Steele, B. Vural, Wave Interactions in Solid-State Plasmas, McGraw-Hill, New York 1969.

[3] A. Singh, Int. J. Electron. 34, 127 (1973).

[4] A.A. Bulgakov, S.I. K'hankina, V.M. Yakovenko, Soviet Phys.-Solid State 17, 1947 (1976).

[5] H.C. Hsiel, J. Appl. Plıys. 45, 489 (1974).

[6] S. Ghosh, V.K. Agarwal, Indian J. Phys. A 50, 55 (1985).

[7] V.V. Pavlovich, E.M. Epslitein, Soviet Plıys.-Semicond. 7, 46 (1974).

[8] M. Dey(Bose), S. Ghosh, Phys. Status Solidi B 159, 817 (1990).

[9] S. Ghosh, M.S. Pathak, Phys. Status Solidi B 129, 81 (1985).

[10] S. Guha, S. Ghosh, J. Phys. Chem. Solids 40, 1143 (1979).

[11] A. Neogi, S. Gliosh, J. Appl. Phys. 69, 61 (1991).

[12] V.N. Belyi, B.B. Sevruk, Zh. Tekhn. Fiz. 57, 336 (1987) (in Russian).

[13] O.L. Artemenko, B.B. Sevruk, Pliys. Status Solidi B 180, 257 (1995).

[14] Yu.I. Sirotin, M.P. Shaskolskaya, Fundamentals of the Physics of Crystals, Nauka, Moskva 1979 (in Russian). 